\title{
Analysis of neurodegenerative disease- causing genes in dementia with Lewy bodies
}

\author{
Tatiana Orme ${ }^{1}$, Dena Hernandez ${ }^{2}$, Owen A. Ross ${ }^{3}$, Celia Kun-Rodrigues ${ }^{4}$, Lee Darwent ${ }^{1}$, Claire E. Shepherd ${ }^{5}$, \\ Laura Parkkinen ${ }^{6}$, Olaf Ansorge ${ }^{6}$, Lorraine Clark ${ }^{7}$, Lawrence S. Honig ${ }^{7}$, Karen Marder $^{7}$, Afina Lemstra ${ }^{8}$, \\ Ekaterina Rogaeva ${ }^{9}$, Peter St. George-Hyslop ${ }^{9}$, Elisabet Londos ${ }^{10}$, Henrik Zetterberg ${ }^{11,12}$, Kevin Morgan ${ }^{13}$, \\ Claire Troakes $^{14}$, Safa Al-Sarraj ${ }^{14}$, Tammaryn Lashley ${ }^{15}$, Janice Holton ${ }^{15}$, Yaroslau Compta ${ }^{15,16}$, \\ Vivianna Van Deerlin ${ }^{17}$, John Q. Trojanowski ${ }^{17}$, Geidy E. Serrano ${ }^{18}$, Thomas G. Beach ${ }^{18}$, Suzanne Lesage ${ }^{19,20}$ \\ Douglas Galasko ${ }^{20}$, Eliezer Masliah ${ }^{21}$, Isabel Santana ${ }^{22}$, Pau Pastor ${ }^{23,24}$, Pentti J. Tienari ${ }^{25}$, Liisa Myllykangas ${ }^{26}$, \\ Minna Oinas ${ }^{27}$, Tamas Revesz ${ }^{15}$, Andrew Lees ${ }^{15}$, Brad F. Boeve ${ }^{28}$, Ronald C. Petersen ${ }^{29}$, Tanis J. Ferman ${ }^{29}$, \\ Valentina Escott-Price ${ }^{30}$, Neill Graff-Radford ${ }^{31}$, Nigel J. Cairns ${ }^{32}$, John C. Morris ${ }^{33}$, Stuart Pickering-Brown ${ }^{33}$, \\ David Mann ${ }^{33}$, Glenda Halliday ${ }^{5}$, David J. Stone ${ }^{34}$, Dennis W. Dickson ${ }^{3}$, John Hardy ${ }^{1}$, Andrew Singleton², \\ Rita Guerreiro ${ }^{4}$ and Jose Bras ${ }^{4 *}$ (D)
}

\begin{abstract}
Dementia with Lewy bodies (DLB) is a clinically heterogeneous disorder with a substantial burden on healthcare. Despite this, the genetic basis of the disorder is not well defined and its boundaries with other neurodegenerative diseases are unclear. Here, we performed whole exome sequencing of a cohort of 1118 Caucasian DLB patients, and focused on genes causative of monogenic neurodegenerative diseases. We analyzed variants in 60 genes implicated in DLB, Alzheimer's disease, Parkinson's disease, frontotemporal dementia, and atypical parkinsonian or dementia disorders, in order to determine their frequency in DLB. We focused on variants that have previously been reported as pathogenic, and also describe variants reported as pathogenic which remain of unknown clinical significance, as well as variants associated with strong risk. Rare missense variants of unknown significance were found in APP, CHCHD2, DCTN1, GRN, MAPT, NOTCH3, SQSTM1, TBK1 and TIA1. Additionally, we identified a pathogenic GRN p.Arg493* mutation, potentially adding to the diversity of phenotypes associated with this mutation. The rarity of previously reported pathogenic mutations in this cohort suggests that the genetic overlap of other neurodegenerative diseases with DLB is not substantial. Since it is now clear that genetics plays a role in DLB, these data suggest that other genetic loci play a role in this disease.
\end{abstract}

\section{Introduction}

Dementia with Lewy bodies (DLB) is a neurodegenerative disease that shares clinical and pathological features with both Parkinson's disease (PD) and Alzheimer's disease (AD). A disease most often occurring in the elderly demographic, it exhibits a varied clinical presentation that can include dementia, parkinsonism, visual hallucinations, REM

\footnotetext{
* Correspondence: Jose.Bras@vai.org

${ }^{4}$ Center for Neurodegenerative Science, Van Andel Research Institute, Grand Rapids, Michigan, USA

Full list of author information is available at the end of the article
}

sleep behavior disorder, fluctuations in attention and alertness, as well as autonomic and psychiatric dysfunction [49], all described as clinical features of underlying synucleinopathy. Similar to PD, the pathological hallmark of DLB is accumulation of alpha-synuclein in Lewy bodies (LBs) and Lewy neurites, leading to consideration of these conditions as different ends of the same clinico-pathological spectrum. Lewy-related pathology in DLB can be found not only in the brainstem, but also widespread in limbic and/or neocortical regions [47]. Alzheimer pathology in the form of

(C) The Author(s). 2020 Open Access This article is distributed under the terms of the Creative Commons Attribution 4.0 International License (http://creativecommons.org/licenses/by/4.0/), which permits unrestricted use, distribution, and 
amyloid plaques and tau neurofibrillary tangles are frequently found at autopsy [17].

At present, knowledge of the genetic etiology of DLB is limited. Families with the disorder are rare, and $S N C A$ multiplications and point mutations have been shown to cause disease in multiplex families of mixed Parkinson's disease and dementia [31, 63, 77]. Variants in PRNP [38] and $S N C B$ [55] have been reported in DLB, but their pathogenicity has been questioned due to lack of replication, lack of segregation [55], or presence in healthy controls [8]. DLB shares risk loci that are associated with $\mathrm{AD}$ or $\mathrm{PD}$, and we have recently shown the genetic correlation between DLB and PD, and DLB and AD, is approximately equal when disregarding $A P O E$ [26]. The $A P O E \& 4$ allele strongly predisposes to the development of the disorder [13, 57, 64, 69], as do certain variants in the GBA gene [53]. A non-synonymous variant in PLCG2 has been proposed to confer protection from $A D$ [62], and more recently, DLB and frontotemporal dementia (FTD) [71]. An association at the SNCA locus was identified, which, interestingly, shows a differential association profile in DLB than that seen in PD [13]. Furthermore, the first genome-wide association study (GWAS) was recently published, analyzing the genotyping data from 1743 DLB patients, a proportion of whom (745 samples) are also included in this study. In a two-stage study design, the associations at $A P O E, G B A$ and $S N C A$ were replicated, and novel loci reached suggestive levels of association [27]. It has been proposed that rare variants in $\mathrm{AD}$ and $\mathrm{PD}$ causing genes may also play a role in sporadic DLB. However, these studies have been small, with sample sizes of approximately 100 cases [36, 37, 51]; it is thus uncertain whether these findings are merely coincidental.

We performed exome sequencing of over 1000 DLB patients to investigate the role of genetic variants in not only $\mathrm{AD}$ and $\mathrm{PD}$ genes, but additionally in a collection of approximately 40 disease genes established as causative of neurodegenerative diseases. The genetic variants studied were those previously implicated in FTD with or without amyotrophic lateral sclerosis (ALS); atypical parkinsonian disorders; and disorders with dementia as a presenting feature.

As DLB is a heterogeneous disorder, and has overlapping features with other diseases, accurate diagnosis relies on the combination of clinical and pathological assessments. To mitigate this issue as much as possible, the DLB patients included in this study had a neuropathological diagnosis of DLB.

\section{Methods}

\section{Cohort studied}

We studied 1118 patients neuropathologically diagnosed according to the 2005 McKeith diagnosis guidelines [49], as meeting the criteria for 'intermediate' or 'high' likelihood DLB and self-reported as Caucasian. The mean age at death in the cohort was $78.8( \pm 8.4)$ years and the male to female ratio was 1.5 .

\section{Exome sequencing and data analysis}

DNA was extracted from the cerebellum or frontal cortex using standard methods [75]. Exome sequencing was performed using Illumina's Nextera Rapid Expanded Exome capture $(62 \mathrm{Mb})$, or Agilent SureSelect Exome Capture Kit (v4) and sequenced on Illumina's HiSeq2500 using $100 \mathrm{bp}$ paired-end reads. On target average coverage of at least 30x was obtained for all included samples. Data analysis was performed according to standard GATK (v3) best practices [50] using a single informatics pipeline and performing joint variant calling of single nucleotide variants (SNVs) and short insertions and deletions (indels) across all samples. In brief, sequencing reads were aligned to the human reference genome (GRCh37/hg19) using bwamem (v0.7.12), duplicate reads were flagged using samblaster (v0.1.21), and realignment around indels and base quality scores were recalibrated using GATK. Variant recalibration was performed using GATK's variant quality score recalibration (VQSR) [21, 70]. Variants that did not meet the VQSR threshold of 99.9 were excluded. Individual genotypes with a phred-scaled quality score below 20 and with coverage below 8 were set to missing. Variants were included only if they had a high call rate in both capture kits (genotyped in $\geq 90 \%$ of samples). Annotation of variants was performed with snpEff (v4.2) [15] and dbNSFP v2.9 [44] using GRCh37/hg19 as reference.

\section{Sample quality control (QC)}

Sample quality control metrics were generated using PLINK 1.9 [58]. Population structure was analysed using principal component analysis, and samples that did not cluster with the European population of the 1000 Genomes dataset were removed from analysis. Concordance between reported and genotype sex was performed for each sample to remove those with a discordant sex assignment. Samples with inconsistent heterozygosity rates, or that were shown to be related or duplicated were excluded. Due to linkage disequilibrium, population structure and genotyping error, we removed an individual from a pair of samples using a proportion of identity by descent threshold of $\geq 0.1875$ [3]. After QC measures, we analyzed variants from 1004 DLB patients. The locations from which the DLB samples were sourced can be found in Additional file 1: Table S1.

\section{Neurodegenerative disease genes}

Fifty-seven genes were selected based on their role in monogenic forms of neurodegenerative diseases including $\mathrm{AD}, \mathrm{PD}, \mathrm{FTD}$ and related Mendelian disorders featuring parkinsonism or dementia. Due to its relevance to DLB, we also report on variants in $G B A$ and $A P O E$, as well as 
the PLCG2 p.Pro522Arg variant. A full list of the studied genes can be found in Additional file 2: Table S2.

We focused on variants that were amino acid changing; or that fell in splice donor/acceptor sites; in splice regions, or in $5^{\prime}$ or $3^{\prime}$ untranslated regions.

A literature search was conducted for each diseasecausing gene studied in order to identify previously reported pathogenic mutations. This included primary literature reports; supplementary information; the Human Gene Mutation Database [66]; the Online Mendelian Inheritance in Man website [2]; the AD \& FTD, and PD mutation databases [20]; and Clinvar [39]. Population variant frequency was determined using gnomAD (v2.1), a genomic database consisting of variants from 125,748 exomes and 15,708 genomes [34, 41].

The maximum frequency that a known pathogenic variant occurs in a large cohort of a disease of interest allows for an estimation of the maximum tolerated frequency of a pathogenic variant for that disease [74]. As the genetics of DLB is yet to be fully delineated, we used Alzheimer's disease to estimate a maximum tolerated allele count for a pathogenic variant to occur in the gnomAD database, implemented using the alleleFrequencyApp http://cardiodb. org/allelefrequencyapp/ [74]. This was used as a conservative approximation of maximum tolerated variant frequencies for pathogenic mutations in the general population for a disease such as AD.

Reported variants were also analysed for their frequency in 432 control individuals from the Healthy Exomes (HEX) dataset, who died aged 60 or over, without any diseaseassociated neuropathology [29, 28].

\section{APOE genotyping}

Given the difficulty in sequencing the $A P O E$ locus, the $A P O E$ haplotype was confirmed in samples where DNA was available ( $n=758$ samples). APOE haplotype genotyping was conducted using enzyme restriction, as previously described [29].

\section{Sanger sequencing}

Candidate variants were confirmed by Sanger sequencing when DNA was available. DNA was amplified by polymerase chain reaction using Roche FastStart PCR Master Mix (Roche Diagnostics Corp) and sequenced with Applied Biosystems BigDye terminator version 3.1 sequencing chemistry in an ABI3730XL genetic analyzer as per manufacturer's instructions (Applied Biosystems). Primers are available upon request. The sequences were analysed using Sequencher software version 4.2 (Gene Codes).

\section{Results}

We performed a detailed analysis of the genetic variability of 57 genes shown previously to cause neurodegenerative diseases in over 1000 DLB cases. Furthermore, we also report on $G B A$ and $A P O E$, well established risk genes for DLB, as well as the recently reported protective PLCG2 p.Pro522Arg variant.

We identified a pathogenic nonsense mutation in GRN, p.Arg493* (ENST00000053867), in one patient. This variant was confirmed by Sanger sequencing. In addition, we identified previously reported mutations that, due to their low frequency in the general population, have uncertain clinical significance (Table 1). No previously reported pathogenic homozygous variants were identified in genes that cause neurodegenerative disease with autosomal recessive inheritance. As the data was not phased, compound heterozygous variants could not be completely assessed.

\section{GRN p.Arg493*}

The patient with the p.Arg493* GRN mutation had a medical history that included a stroke, and previous surgical evacuation of a subdural haematoma, as well as a family history of $\mathrm{AD}$ in a parent and a sibling, with onset in the seventh decade. The presenting symptom was episodes of confusion, and a CT scan showed moderate cerebral and cerebellar atrophy with a lacunar infarct in the left external capsule. Subsequent symptoms included memory loss, disorientation, altered gait, stooped posture, right-sided rigidity and visual hallucinations. Impaired smooth pursuit, left hemiparesis, left spasticity, right-sided rigidity, and a slight parkinsonian tremor of the left hand were also reported. The patient was severely demented and scored $3 / 30$ on the Mini Mental State Examination (MMSE) in an assessment conducted at 83 years, approximately 2 years before death.

Macroscopically, the right convexity of the brain showed very marked asymmetrical atrophy of the frontal, temporal and parietal lobes, with knife-edge atrophy at the frontal and temporal poles, and mild atrophy of the right occipital lobe. There were no focal lesions. Pathological examination with immunohistochemical staining for alpha-synuclein protein showed a single LB in the substantia nigra; very infrequent Lewy bodies in multiple cortical areas; sparse LBs in the anterior cingulate, entorhinal and transentorhinal cortices; and no LBs in the amygdala. Thioflavin S methods showed frequent diffuse amyloid plaques in neocortical areas. Very infrequent neurofibrillary tangles were present in the nucleus basalis of Meynert, hypothalamus and entorhinal cortex, and were absent from all neocortical areas examined. Immunohistochemical staining for abnormal tau protein identified frequent dot-like features and diffusely stained neurons in the entorhinal cortex, transentorhinal cortex, CA1 and subiculum of the hippocampus and the parahippocampal gyrus. There were sparse argyrophilic grains in the amygdala, entorhinal cortex and area CA1 of the subiculum. Immunohistochemical staining for ubiquitin showed moderate densities of short neuropil threads and dots, as well as sparse to moderate densities of small cytoplasmic inclusions in layer II of 


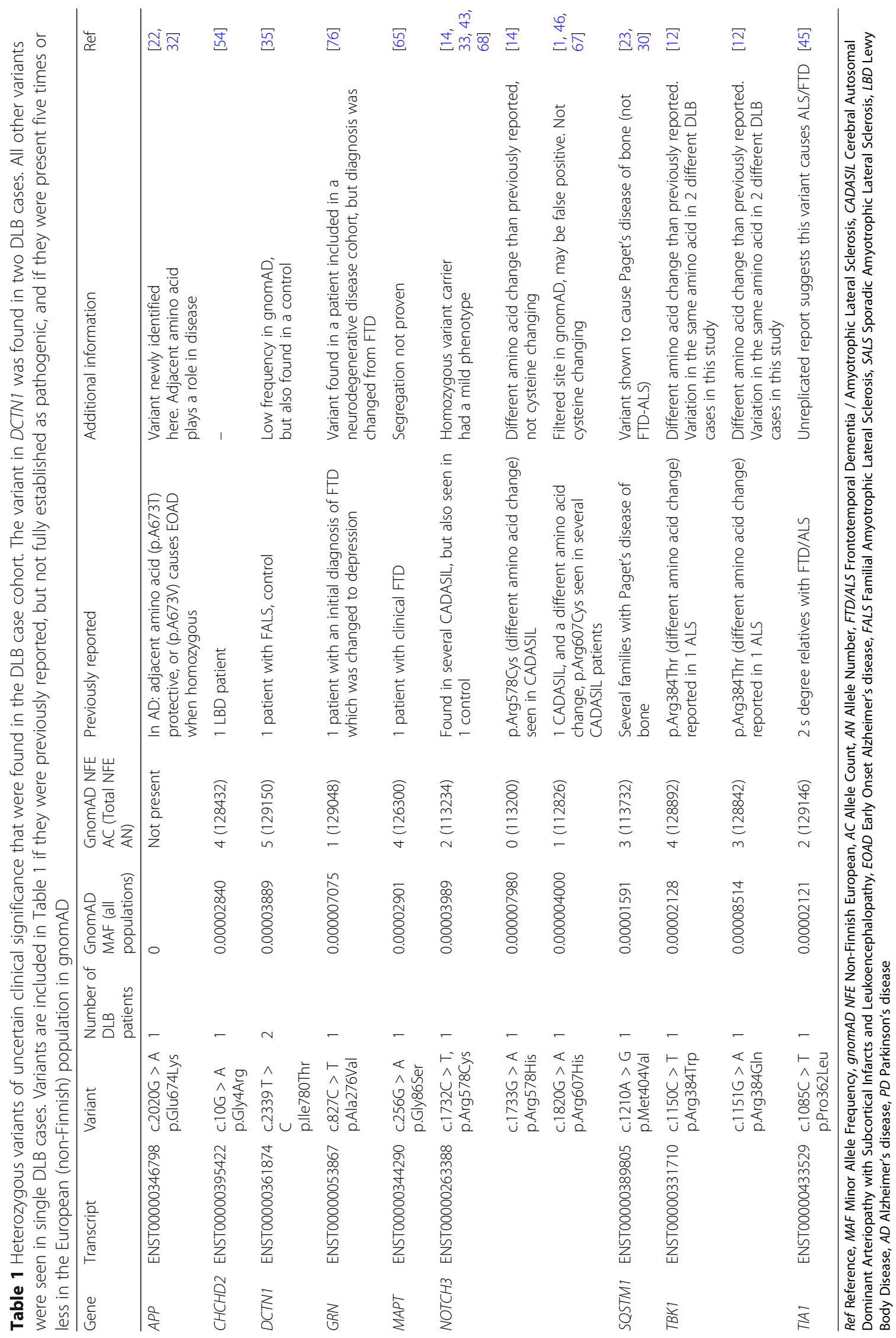


frontal and temporal neocortical regions. Following the identification of the mutation, the sample was stained for TDP-43 and revealed inclusions in frontal and temporal lobes. Sections stained immunohistochemically for phosphorylated TDP-43 protein showed immunoreactive dystrophic puncta, neurites and perikaryal cytoplasmic inclusions within layer II of frontal and temporal neocortical areas, as well as the amygdala, hippocampal CA1 region, and entorhinal cortex. These features reached moderate to frequent densities in the frontal and temporal neocortex.

\section{Established risk factors for DLB}

No homozygous Gaucher disease causing variants were identified in GBA. Table 2 details variants found in $G B A$ that had a call-rate of $90 \%$ or greater. Two variants, p.Glu365Lys and p.Asn409Ser were over-represented in DLB compared to the gnomAD database. Of note, 2 homozygous carriers of p.Glu365Lys were identified and a novel variant, p.Asn409del, was identified in 1 patient in the cohort.

APOE haplotypes were independently genotyped in 758 DLB samples where DNA was available. Of this subset of samples, the $\varepsilon 4$ allele frequency was $48.6 \%$, in keeping with the prevalence of this allele in previous reports $[64,69]$.

\section{Potentially pathogenic variants}

We used Alzheimer's disease genetics as a model in order to apply an approximate threshold for the tolerated occurrence of pathogenic $\mathrm{AD}$ mutations in gnomAD. We applied an AD prevalence of 1/79 (Alzheimer Society Dementia report, 2014); a maximum estimated disease-causing MAF of 0.0012; and a very conservative penetrance threshold of 0.5 . The maximum tolerated frequency for a pathogenic allele for $\mathrm{AD}$ using the allele number of a variant genotyped in the entire gnomAD European (non-Finnish) population was $1.5556 \times 10^{-5}$, or five alleles. This is a very conservative approximation, likely to be too high since $A D$ is more prevalent than DLB, however since the genetic architecture of DLB still remains largely unresolved, we used $A D$ as an approximation. In Table 1 we describe previously reported variants of unknown clinical significance that were present in 5 or less Europeans in the gnomAD database. None of these variants were detected in the control individuals from the HEX dataset. These variants were identified in $A P P$, CHCHD2, DCTN1, GRN, MAPT, NOTCH3, SQSTM1, TBK1 and TIA1. Variants were reported in Table 1 if the evidence for pathogenicity was moderate, such as having unproven segregation with disease; eliciting a different amino acid change than was previously reported; identified in a gene that has not been replicated as causative for disease; or if the variant has also been identified in a control subject. Table 1 also shows variants not present in Europeans in gnomAD that affect the same or an adjacent amino acid as previously reported in disease. Table 3 lists variants identified in multiple DLB cases and with 5 or less alleles in gnomAD.

Variants that have previously been reported in disease, but were more frequent in the general population, thus less likely to be fully penetrant, disease-causing alleles, are reported in Additional file 3: Table S3. These include variants such as SNCA p.His50Gln, which is present in 19 Europeans in gnomAD, and whose pathogenicity has been disputed [10].

\section{Genes previously reported in DLB}

We investigated variants in genes that have been reported as causative of DLB - PRNP and SNCB - as well as a gene in which a variant was recently reported as protective for DLB - PLCG2.

The previously identified PRNP p.Met232Arg [38] variant was not present in our cohort, and there were no other previously reported pathogenic variants in PRNP in our data. It is therefore unlikely that PRNP mutations cause sporadic DLB in the European population.

The $S N C B$ p.Val70Met and p.Pro123His variants reported to predispose to DLB [55] were also not found in our cohort of DLB cases, suggesting that if they play a role in DLB, they may be population specific risk factors. In our cohort, no non-synonymous variants were found in the $S N C B$ gene, even though the entire gene was adequately covered.

A variant in PLCG2, p.Pro522Arg, has been reported to reduce the risk of DLB, AD and FTD [71]. This variant was identified in 18 DLB cases (MAF 0.0089), which is similar to the frequency found in gnomAD non-Finnish Europeans (MAF 0.0087).

\section{TREM2}

TREM2 is the second strongest genetic risk factor for $\mathrm{AD}$, an effect largely driven by the p.R47H variant. In our data, the p.Arg47His variant, which was successfully sequenced in 667 of the 1004 samples, had a MAF 0.00299, which is similar to the frequency in NFEs in gnomAD (MAF 0.002466). The p.Arg62His variant also had a similar MAF in DLB (0.0142), compared to Europeans in the gnomAD database (0.0112). Likewise, the frequency of p.Thr96Lys and p.Leu211Pro variants was similar in DLB patients and gnomAD, (MAF 0.000991 versus 0.00101, and MAF 0.000992 versus 0.00113), respectively. No homozygous variants in TREM2 were identified.

\section{Discussion}

We present a comprehensive analysis of rare genetic variability in an extensive number of neurodegenerative disease-causing genes in a large cohort of patients diagnosed with DLB. We used state-of-the-art analytical approaches with well-established quality control criteria that allowed us to identify genetic variability and estimate its contribution to disease. 


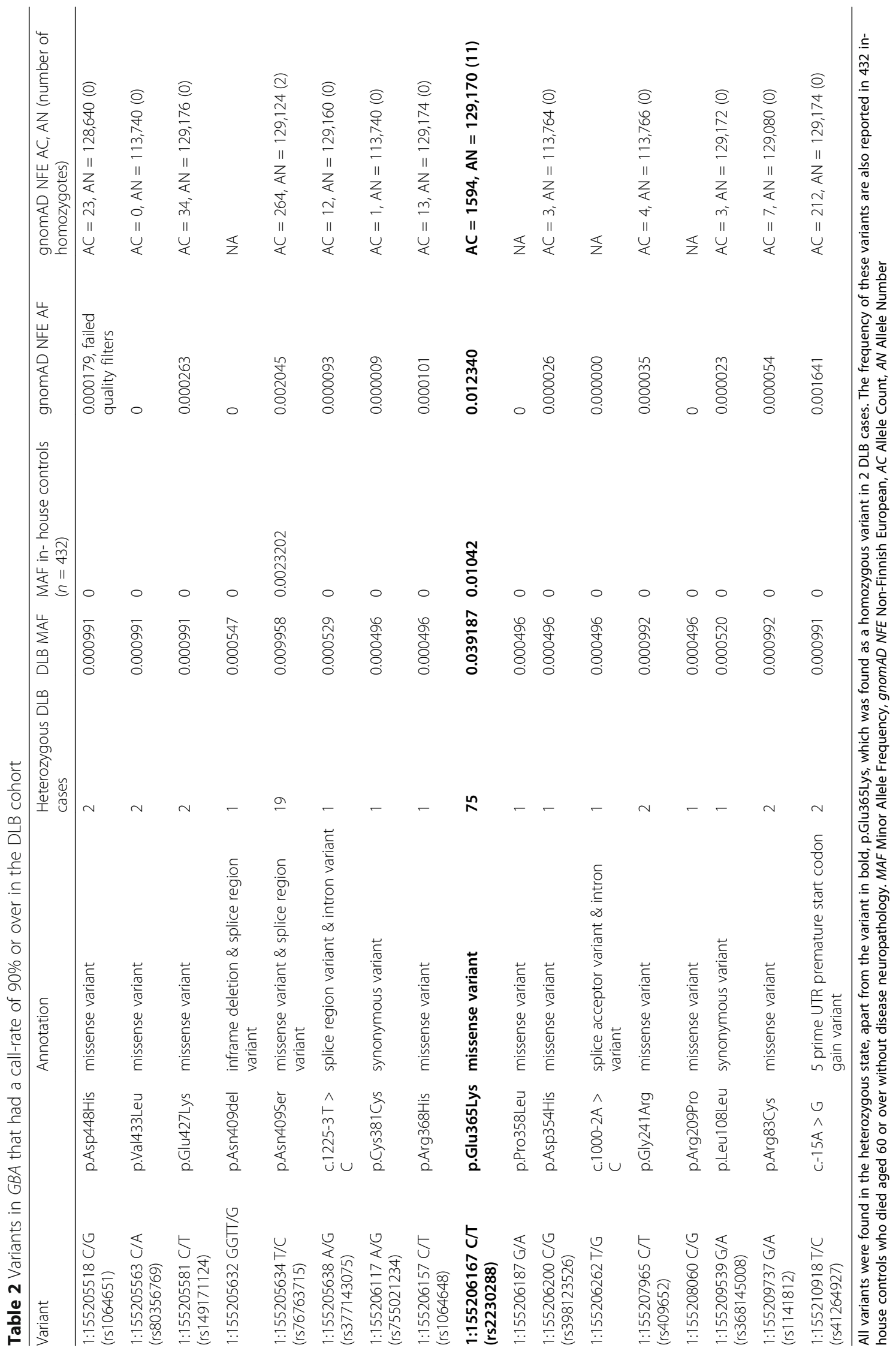


Table 3 Variants found in more than 1 DLB case, that were present in 5 or less individuals from the gnomAD exome dataset of non-Finnish Europeans

\begin{tabular}{lllll}
\hline Gene & Transcript & Variant & Number of DLB cases & GnomAD NFE AC (Total NFE AN) \\
\hline PARK2 & ENST00000366898 & p.Gln440Arg & 2 & $1(112822)$ \\
VPS13C & ENST00000261517 & p.lle288Met & 2 & $4(112094)$ \\
TUBA4A & ENST00000248437 & p.Val181Met & 2 & $5(113764)$ \\
DNAJC6 & ENST00000371069 & p.Val632Ala & 2 & $0(83222)$ \\
VPS35 & ENST00000299138 & p.Arg499His & 2 & $4(113646)$ \\
\hline
\end{tabular}

All variants were identified in the heterozygous state. gnomAD NFE Non-Finnish European, AC Allele Count, AN Allele Number

Mutations in GRN cause FTD [7, 19], and the most commonly reported pathogenic mutation in GRN is p.Arg493* [59]. The patient described here lacked prominent clinical signs of FTD, such as changes in behavior, personality, or language impairment. Severe dementia, parkinsonism and visual hallucinations were present, and led to the suggested clinical diagnosis of Alzheimer's disease or mixed vascular dementia, with a final neuropathological diagnosis of Alzheimer's disease, dementia with Lewy bodies and argyrophilic grain disease. Substantial phenotypic variability has been described in patients with a GRN p.Arg493* mutation. In 34 patients identified with this mutation, 25 had a diagnosis of FTD; 4 of primary progressive aphasia; 3 of corticobasal syndrome and 3 of Alzheimer's disease. Age at onset ranged from 44 to 69 , and the most common initial symptom was a change in personality or executive dysfunction (25/33 patients), with subsequent language impairment occurring in 26 of 33 patients. Fourteen out of thirty patients had parkinsonism and $10 \%$ had visual hallucinations [59]. This mutation has also been found in 4 patients with sporadic Alzheimer's disease [24].

An overlap between FTD and DLB has been shown both clinically and neuropathologically. Several patients have been identified that simultaneously met clinical criteria for both FTD and DLB $[16,52]$, or that presented clinically with FTD, but at autopsy had DLB [11, 61]. Concomitant TDP-43 and alpha-synuclein pathology can be found in $27-60 \%$ of DLB cases at autopsy [4, 17, 48]. In general, GRN mutations result in disease with clinical heterogeneity $[40,72,73]$, and it is possible that overlaps between DLB and FTD may be connected to variation in GRN. It is also possible that hallucinations and delusions in GRN carriers may cause a misdiagnosis of DLB [9]. An FTD case harboring the GRN p.Thr382fs mutation showed a phenotype resembling DLB, with fluctuations in cognition, parkinsonism and visual hallucinations [5]. Furthermore, alpha-synuclein and TDP-43 pathologies were found in a subset of brains of patients with a GRN mutation [42]. A small study in 58 DLB cases showed that together, rare variants in GRN are associated with DLB [37], although this is yet to be independently replicated. A recent study identified 9 heterogeneous FTLD cases with coexisting Lewy body pathology Braak stage $\geq \mathrm{IV}$, comprising $7 \%$ of the cohort. Two of the 9 cases had a secondary diagnosis of DLB, and a further 2 had GRN mutations [25]. Progranulin and $\beta$-amyloid have been shown to co-localize in plaques in DLB, suggesting a possible biological association between these two aggregated proteins [60]. However, there are often multiple concomitant pathologies that are identified in neurodegenerative disease, and so the observation of TDP-43 with Lewy and amyloid pathology could simply be coincidental multi-morbidity of simultaneous pathologies that coexist in the ageing brain [6]. After the identification of the GRN mutation, additional clinicopathological information was reviewed to assess this case. The histopathological findings of the p.Arg493* carrier revealed Lewy bodies; amyloid plaques; argyrophilic grains; ubiquitin positive inclusions; tau staining, and when reanalysed, TDP-43 pathology. The medical history reported the occurrence of a stroke, which was also confirmed by a CT scan, however no further clinical information was available. Taken together, these facts add to the difficulty in classifying these more complex forms of disease. Particularly in archive cases where the neuropathology assessment did not originally include staining for TDP-43.

We identified possible pathogenic variants in other genes linked to the FTD-ALS spectrum of disease. A variant in TIA1, p.Pro362Leu, suggested to be causative of ALS/FTD, was found in 1 DLB patient in the cohort. TIA1 encodes an RNA-binding protein, and this variant was reported in a pair of second degree relatives with ALS/FTD, as well as a clinically symptomatic, but non-demented relative. An increased burden of rare heterozygous TIA1 mutations in a larger ALS/FTD cohort was reported [45]. In two neuropathologically diagnosed DLB cases, we found two variants in TBK1 that affected the same amino acid: p.Arg384Trp and p.Arg384Gln. This amino acid has been found to be altered in a sporadic Sardinian ALS case (p.Arg384Thr) [12]: a variant not present in gnom $A D$, but whose pathogenicity is unconfirmed. It is intriguing that two DLB cases were identified with variants that affect the same amino acid. 
These variants are present in gnomAD (MAFs 0.0000213 and 0.0000851 , respectively). At present, their contribution to DLB is unclear.

From the perspective of $\mathrm{AD}$ related genetics, we identified a variant of unknown significance in APP - p.Glu674Lys in a DLB case, a substitution of a negatively charged amino acid to a positively charged amino acid at the 3rd residue of the $\beta$-amyloid peptide. This variant was not present in the gnomAD database, and affects an adjacent amino acid to that previously described. The p.Ala673Val was shown to cause early onset Alzheimer's disease in the homozygous state, whereas heterozygous individuals in the family were unaffected [22]. A different amino acid change at the same position, p.Ala673Thr, has been reported in a patient without $\mathrm{AD}$ [56], and predicted as non pathogenic due to non segregation with disease in a family [18]. Furthermore, this variant was shown to be protective against Alzheimer's disease in the Icelandic population, where it resulted in a reduction of beta-secretase cleavage in vitro [32].

We have also looked at strong risk modulating genes: GBA, TREM2, APOE and PLCG2. If at GBA and APOE we saw evidence of increased frequency in the previously reported variants, we did not observe such a finding at TREM2 or PLCG2, with both showing frequencies that are identical to those found in the general population.

We have also identified a number of possible pathogenic mutations in several other genes studied here (Table 1). The design of this study does not allow variant pathogenicity to be unequivocally established, and, in this way, we report these variants to allow future studies to attempt confirmation of these findings.

In summary, we provide the first large-scale characterization of rare genetic variability in the most relevant neurodegenerative disease-causing genes in DLB. Our findings suggest that mutations in genes known to cause other neurodegenerative diseases are not a common cause of DLB.

\section{Supplementary information}

Supplementary information accompanies this paper at https://doi.org/10. 1186/s40478-020-0879-z.

Additional file 1: Table S1. Sources of samples. Research groups, clinical teams and brain banks where the DLB samples included in this study were collected from.

Additional file 2: Table S2. Neurodegenerative disease-causing genes and DLB risk genes analysed in this study. Genes known to cause neurodegenerative diseases are presented according to the mode of inheritance of the respective mendelian disease. Genes such as PARK2, FBXO7, SYNJ1, and DNAJC6, among others, are commonly referred to as parkinson's disease genes, although the clinical and pathological characteristics may be atypical in some cases. FTD/ALS - frontotemporal dementia/ Amyotrophic lateral sclerosis, CADASIL - Cerebral arteriopathy, autosomal dominant, with subcortical infarcts and leukoencephalopathy, CARASIL Cerebral arteriopathy, autosomal recessive, with subcortical infarcts and leukoencephalopathy *Both TMEM230 and DNAJC13 have been hypothesised to be the cause of Parkinson's disease in the same family.
Additional file 3: Table S3. Variants identified in the studied DLB cohort that have been previously reported in disease and have a gnomAD european allele count $>5$. GnomAD NFE AC $=$ gnomAD nonFinnish European allele count. GnomAD NFE AN = gnomAD non-Finnish European allele number. GnomAD Total MAF = gnomAD all populations minor allele frequency.

\section{Acknowledgements}

This work was supported in part by the National Institutes of Neurological Disease and Stroke and by the Alzheimer's Society. TO was supported by a scholarship from the Lewy Body Society. We would like to thank Dr. Daniela Hansen from the Reta Lila Weston Institute at the Institute of Neurology, UCL. For the neuropathologically confirmed samples from Australia, tissues were received from the Sydney Brain Bank which is supported by Neuroscience Research Australia and the University of New South Wales. We acknowledge the Oxford Brain Bank, supported by the Medical Research Council (MRC), Brains for Dementia Research (BDR) (Alzheimer Society and Alzheimer Research UK), Autistica UK and the NIHR Oxford Biomedical Research Centre. We would like to thank the South West Dementia Brain Bank (SWDBB) for providing brain tissue for this study. The SWDBB is part of the Brains for Dementia Research programme, jointly funded by Alzheimer's Research UK and Alzheimer's Society and is supported by BRACE (Bristol Research into Alzheimer's and Care of the Elderly) and the Medical Research Council. Regarding the brain samples and/or bio samples that were obtained from The Netherlands Brain Bank, Netherlands Institute for Neuroscience, Amsterdam (open access: www.brainbank.nl): all Material has been collected from donors for or from whom a written informed consent for a brain autopsy and the use of the material and clinical information for research purposes had been obtained by the NBB. This study was also partially funded by the Canadian Consortium on Neurodegeneration in Aging (Rogaeva and St. George-Hyslop). The Nottingham Genetics Group is supported by ARUK and The Big Lottery Fund. The effort from Columbia University was supported by the Taub Institute, the Panasci Fund, the Parkinson's Disease Foundation, and NIH grants NS060113 (L. Clark), P50AG008702 (P.I. Scott Small), P50NS038370 (P.I. R. Burke), and UL1TR000040 (P.I. H. Ginsberg). O.A.R. is supported by the Michael J. Fox Foundation, NINDS R01\# NS078086. The Mayo Clinic Jacksonville is a Morris K. Udall Parkinson's Disease Research Center of Excellence (NINDS P50 \#NS072187) and is supported by The Little Family Foundation and by the Mangurian Foundation Program for Lewy Body Dementia research and the Alzheimer Disease Research Center (P50 AG016547). The Brain and Body Donation Program (TGB) has been supported by the National Institute of Neurological Disorders and Stroke (U24 NS072026 National Brain and Tissue Resource for Parkinson's Disease and Related Disorders), the National Institute on Aging (P30 AG19610 Arizona Alzheimer's Disease Core Center), the Arizona Department of Health Services (contract 211002, Arizona Alzheimer's Research Center), the Arizona Biomedical Research Commission (contracts 4001, 0011, 05-901 and 1001 to the Arizona Parkinson's Disease Consortium) and the Michael J. Fox Foundation for Parkinson's Research. The work from the Mayo Clinic Rochester is supported by the National Institute on Aging (P50 AG016574 and U01 AG006786). This work has received support from The Queen Square Brain Bank at the UCL Institute of Neurology; where TL is funded by an ARUK senior fellowship. Some of the tissue samples studied were provided by the MRC London Neurodegenerative Diseases Brain Bank and the Brains for Dementia Research project (funded by Alzheimer's Society and ARUK). This research was supported in part by both the NIHR UCLH Biomedical Research Centre and the Queen Square Dementia Biomedical Research Unit. This work was supported in part by the Intramural Research Program of the National Institute on Aging, National Institutes of Health, Department of Health and Human Services; project AG000951-12. The University of Pennsylvania case collection is funded by the Penn Alzheimer's Disease Core Center (AG10124) and the Penn Morris K. Udall Parkinson's Disease Research Center (NS053488). The authors would like to thank the Genome Aggregation Database and the groups that provided exome and genome variant data for comparison. A full list of contributing groups can be found at https://gnomad.broadinstitute.org/. Tissue samples from UCSD are supported by NIH grant AG05131. The authors thank the brain bank GIE NeuroCEB, the French program "Investissements d'avenir" (ANR-10-IAIHU-06). PJT and LM are supported by the Helsinki University Central Hospital, the Sigrid Juselius Foundation, the Folkhälsan Research Foundation and the Finnish Academy. $\mathrm{HZ}$ is a Wallenberg Academy Fellow and is 
further supported by the UK Dementia Research Institute at UCL, the European Research Council and the Swedish Research Council.

\section{Authors' contributions}

$J B$ and AS conceived the study. JB, AS, RG and TO contributed to study design. JB, DH, CKR and AS contributed to data generation. JB and TO contributed to data analysis. JB, RG and TO contributed to data interpretation. TO drafted the manuscript and JB and RG performed critical review and additional writing. All other authors contributed samples. All authors read and approved the final manuscript.

\section{Competing interests}

The authors declare that they have no competing interests.

\section{Author details}

${ }^{1}$ UK Dementia Research Institute at UCL and Department of Molecular Neuroscience, Institute of Neurology, University College London, London, UK. ${ }^{2}$ Laboratory of Neurogenetics, National Institutes on Aging, $\mathrm{NIH}$, Bethesda, MD, USA. ${ }^{3}$ Department of Neuroscience, Mayo Clinic, Jacksonville, FL, USA. ${ }^{4}$ Center for Neurodegenerative Science, Van Andel Research Institute, Grand Rapids, Michigan, USA. ${ }^{5}$ Neuroscience Research Australia, Sydney, Australia and School of Medical Sciences, Faculty of Medicine, University of New South Wales, Sydney, Australia. ${ }^{6}$ Nuffield Department of Clinical Neurosciences, Oxford Parkinson's Disease Centre, University of Oxford, Oxford, UK. ${ }^{7}$ Taub Institute for Alzheimer Disease and the Aging Brain and Department of Pathology and Cell Biology and Neurology, Columbia University Medical Center, New York, NY, USA. ${ }^{8}$ Department of Neurology and Alzheimer Center, Neuroscience Campus Amsterdam, VU University Medical Center, Amsterdam, the Netherlands. 'Department of Medicine, Tanz Centre for Research in Neurodegenerative Diseases, University of Toronto, Toronto, ON, Canada. ${ }^{10} \mathrm{Clinical}$ Memory Research Unit, Institution of Clinical Sciences Malmö, Lund University, Lund, Sweden. ${ }^{11}$ UK Dementia Research Institute at UCL and Department of Molecular Neuroscience, Institute of Neurology, University College London, London, UK. ${ }^{12}$ Clinical Neurochemistry Laboratory, Institute of Neuroscience and Physiology, Sahlgrenska Academy at the University of Gothenburg, Mölndal, Sweden. ${ }^{13}$ Human Genetics, School of Life Sciences, University of Nottingham, Nottingham, UK. ${ }^{14}$ Department of Basic and Clinical Neuroscience and Institute of Psychiatry, Psychology and Neuroscience, King's College London, London, UK. ${ }^{15}$ Queen Square Brain Bank, Department of Molecular Neuroscience, UCL Institute of Neurology, London, UK. ${ }^{16}$ Parkinson's Disease \& Movement Disorders Unit, Neurology Service, Clinical Neuroscience Institute (ICN), Hospital Clínic, Institut de Neurociències, University Universitat de Barcelona, IDIBAPS, Barcelona, Catalonia, Spain. ${ }^{17}$ Department of Pathology and Laboratory Medicine, Center for Neurodegenerative Disease Research, Perelman School of Medicine at the University of Pennsylvania, 3600 Spruce Street, Philadelphia, USA. ${ }^{18}$ Banner Sun Health Research Institute, 10515 W Santa Fe Drive, Sun City, AZ 85351, USA. ${ }^{19}$ Sorbonne Université, Université Pierre et Marie Curie-Paris 06, Inserm, Centre National de la Reserche Scientifique, and Institute du Cerveau et de la Moelle épinière, Paris, France. ${ }^{20}$ Assistance Publique Hôpitaux de Paris, Hôpital de la Salpêtrière, Département de Génétique et Cytogénétique, Paris, France. ${ }^{21}$ Division of Neurosciences and Laboratory of Neurogenetics, National Institute on Aging/NIH, Bethesda, MD, USA. ${ }^{22}$ Neurology Service, University of Coimbra Hospital, Coimbra, Portugal. ${ }^{23}$ Memory Unit, Department of Neurology, University Hospital Mutua de Terrassa, University of Barcelona, Terrassa, Barcelona, Spain. ${ }^{24}$ Centro de Investigación Biomédica en Red Enfermedades Neurodegenerativas (CIBERNED), Madrid, Spain. ${ }^{25}$ Translational Immunology, Research Programs Unit, Department of Neurology, Helsinki University Hospital, University of Helsinki, Helsinki, Finland. ${ }^{26}$ Department of Pathology, University of Helsinki and HUSLAB, Helsinki University Hospital, Helsinki, Finland. ${ }^{27}$ Department of Neuropathology and Neurosurgery, Helsinki University Hospital and University of Helsinki, Helsinki, Finland. ${ }^{28}$ Neurology Department, Mayo Clinic, Rochester, MN, USA. ${ }^{29}$ Department of Psychiatry and Department of Psychology, Mayo Clinic, Jacksonville, FL, USA. ${ }^{30}$ UK Dementia Research Institute at Cardiff and MRC Centre for Neuropsychiatric Genetics and Genomics, School of Medicine, Cardiff University, Cardiff, UK. ${ }^{31}$ Department of Neurology, Mayo Clinic, Jacksonville, FL, USA. ${ }^{32}$ Department of Neurology, Washington University School of Medicine, Saint Louis, MO, USA. ${ }^{33}$ Division of Neuroscience and Experimental Psychology, Faculty of Biology, Medicine and Health, University of Manchester, Manchester, UK. ${ }^{34}$ Genetics and Pharmacogenomics, Merck \& Co., Inc., West Point, PA, USA.

Received: 20 November 2019 Accepted: 3 January 2020 Published online: 29 January 2020

\section{References}

1. Adib-Samii P, Brice G, Martin RJ, Markus HS (2010) Clinical spectrum of CADASIL and the effect of cardiovascular risk factors on phenotype: study in 200 consecutively recruited individuals. Stroke. 41:630-634

2. Amberger J, Bocchini CA, Scott AF, Hamosh A (2009) McKusick's Online Mendelian Inheritance in Man (OMIM $\left.{ }^{\circledast}\right)$. Nucleic Acids Res 37:D793-D796

3. Anderson CA, Pettersson FH, Clarke GM, Cardon LR, Morris AP, Zondervan KT (2010) Data quality control in genetic case-control association studies. Nat Protoc. 5:1564-1573

4. Arai T, Mackenzie IRA, Hasegawa M, Nonoka T, Niizato K, Tsuchiya K et al (2009) Phosphorylated TDP-43 in Alzheimer's disease and dementia with Lewy bodies. Acta Neuropathol. 117:125-136

5. Arosio B, Abbate C, Galimberti D, Rossi PD, Inglese S, Fenoglio C et al (2013) GRN Thr272fs clinical heterogeneity: a case with atypical late onset presenting with a dementia with Lewy bodies phenotype. J Alzheimers Dis. 35:669-674

6. Attems J (2017) The multi-morbid old brain. Acta Neuropathol. 134:169-170

7. Baker M, Mackenzie IR, Pickering-Brown SM, Gass J, Rademakers R, Lindholm C et al (2006) Mutations in progranulin cause tau-negative frontotemporal dementia linked to chromosome 17. Nature. 442:916-919

8. Beck J, Collinge J, Mead S (2012) Prion protein gene M232R variation is probably an uncommon polymorphism rather than a pathogenic mutation. Brain 135(Pt 2):e209 author reply e210

9. Benussi A, Padovani A, Borroni B (2015) Phenotypic Heterogeneity of Monogenic Frontotemporal Dementia. Front Aging Neurosci. 7:171

10. Blauwendraat C, Kia DA, Pihlstrøm L, Gan-Or Z, Lesage S, Gibbs JR et al (2018) Insufficient evidence for pathogenicity of SNCA His50GIn (H50Q) in Parkinson's disease. Neurobiol Aging 64:159.e5-159.e8

11. Bonner LT, Tsuang DW, Cherrier MM, Eugenio CJ, Du Jennifer Q, Steinbart E et al (2003) Familial dementia with Lewy bodies with an atypical clinical presentation. J Geriatr Psychiatry Neurol. 16:59-64

12. Borghero G, Pugliatti M, Marrosu F, Marrosu MG, Murru MR, Floris G et al (2016) TBK1 is associated with ALS and ALS-FTD in Sardinian patients. Neurobiol Aging 43:180.e1-180.e5

13. Bras J, Guerreiro R, Darwent L, Parkkinen L, Ansorge O, Escott-Price $V$ et al (2014) Genetic analysis implicates APOE, SNCA and suggests lysosomal dysfunction in the etiology of dementia with Lewy bodies. Hum Mol Genet 23:6139-6146

14. Choi JC, Kang S-Y, Kang J-H, Park J-K (2006) Intracerebral hemorrhages in CADASIL. Neurology. 67:2042-2044

15. Cingolani P, Platts A, Wang LL, Coon M, Nguyen T, Wang $L$ et al (2012) A program for annotating and predicting the effects of single nucleotide polymorphisms, SnpEff: SNPs in the genome of Drosophila melanogaster strain w1118; iso-2; iso-3. Fly 6:80-92

16. Claassen DO, Parisi JE, Giannini C, Boeve BF, Dickson DW, Josephs KA (2008) Frontotemporal dementia mimicking dementia with Lewy bodies. Cogn Behav Neurol. 21:157-163

17. Colom-Cadena M, Gelpi E, Charif S, Belbin O, Blesa R, Martí MJ et al (2013) Confluence of a-synuclein, tau, and $\beta$-amyloid pathologies in dementia with Lewy bodies. J Neuropathol Exp Neurol. 72:1203-1212

18. Cruchaga C, Haller G, Chakraverty S, Mayo K, Vallania FLM, Mitra RD et al (2012) Rare variants in APP, PSEN1 and PSEN2 increase risk for AD in lateonset Alzheimer's disease families. PLoS One. 7:e31039

19. Cruts M, Gijselinck I, van der Zee J, Engelborghs S, Wils H, Pirici D et al (2006) Null mutations in progranulin cause ubiquitin-positive frontotemporal dementia linked to chromosome 17q21. Nature. 442:920-924

20. Cruts M, Theuns J, Van Broeckhoven C (2012) Locus-specific mutation databases for neurodegenerative brain diseases. Hum Mutat. 33:1340-1344

21. DePristo MA, Banks E, Poplin R, Garimella KV, Maguire JR, Hartl C et al (2011) A framework for variation discovery and genotyping using next-generation DNA sequencing data. Nat Genet. 43:491-498

22. Di Fede G, Catania M, Morbin M, Rossi G, Suardi S, Mazzoleni G et al (2009) A recessive mutation in the APP gene with dominant-negative effect on amyloidogenesis. Science. 323:1473-1477 
23. Falchetti A, Di Stefano M, Marini F, Del Monte F, Gozzini A, Masi L et al (2005) Segregation of a M404V mutation of the p62/sequestosome 1 (p62/ SQSTM1) gene with polyostotic Paget's disease of bone in an Italian family. Arthritis Res Ther. 7:R1289-R1295

24. Fernández MV, Kim JH, Budde JP, Black K, Medvedeva A, Saef B et al (2017) Analysis of neurodegenerative Mendelian genes in clinically diagnosed Alzheimer Disease. PLoS Genet. 13:e1007045

25. Forrest SL, Crockford DR, Sizemova A, McCann H, Shepherd CE, McGeachie $A B$ et al (2019) Coexisting Lewy body disease and clinical parkinsonism in frontotemporal lobar degeneration. Neurology Available from: https://doi. org/10.1212/WNL.0000000000007530

26. Guerreiro R, Escott-Price V, Darwent L, Parkkinen L, Ansorge O, Hernandez DG et al (2016) Genome-wide analysis of genetic correlation in dementia with Lewy bodies, Parkinson's and Alzheimer's diseases. Neurobiol Aging 38: 214.e7-214.e10

27. Guerreiro R, Ross OA, Kun-Rodrigues C, Hernandez DG, Orme T, Eicher JD et al (2018) Investigating the genetic architecture of dementia with Lewy bodies: a two-stage genome-wide association study. Lancet Neurol. 17:64-74

28. Guerreiro R, Sassi C, Gibbs JR, Edsall C, Hernandez D, Brown K, et al. A comprehensive assessment of benign genetic variability for neurodegenerative disorders. bioRxiv, 2018.

29. Hixson JE, Vernier DT (1990) Restriction isotyping of human apolipoprotein E by gene amplification and cleavage with Hhal. J Lipid Res. 31:545-548

30. Hocking LJ, Lucas GJA, Daroszewska A, Cundy T, Nicholson GC, Donath J et al (2004) Novel UBA domain mutations of SQSTM1 in Paget's disease of bone: genotype phenotype correlation, functional analysis, and structural consequences. J Bone Miner Res. 19:1122-1127

31. Ikeuchi T, Kakita A, Shiga A, Kasuga K, Kaneko H, Tan C-F et al (2008) Patients homozygous and heterozygous for SNCA duplication in a family with parkinsonism and dementia. Arch Neurol. 65:514-519

32. Jonsson T, Atwal JK, Steinberg S, Snaedal J, Jonsson PV, Bjornsson S et al (2012) A mutation in APP protects against Alzheimer's disease and agerelated cognitive decline. Nature. 488:96-99

33. Joutel A, Vahedi $K$, Corpechot C, Troesch A, Chabriat H, Vayssière C et al (1997) Strong clustering and stereotyped nature of Notch3 mutations in CADASIL patients. Lancet. 350:1511-1515

34. Karczewski KJ, Francioli LC, Tiao G, Cummings BB (2019) Variation across 141,456 human exomes and genomes reveals the spectrum of loss-offunction intolerance across human protein-coding genes. BioRxiv Available from: https://www.biorxiv.org/content/10.1101/531210v2.abstract

35. Kenna KP, McLaughlin RL, Byrne S, Elamin M, Heverin M, Kenny EM et al (2013) Delineating the genetic heterogeneity of ALS using targeted highthroughput sequencing. J Med Genet. 50:776-783

36. Keogh MJ, Kurzawa-Akanbi M, Griffin H, Douroudis K, Ayers KL, Hussein Rl et al (2016) Exome sequencing in dementia with Lewy bodies. Transl Psychiatry. 6:e728

37. Keogh MJ, Wei W, Wilson I, Coxhead J, Ryan S, Rollinson S et al (2017) Genetic compendium of 1511 human brains available through the UK Medical Research Council Brain Banks Network Resource. Genome Res. 27:165-173

38. Koide T, Ohtake H, Nakajima T, Furukawa H, Sakai K, Kamei H et al (2002) A patient with dementia with Lewy bodies and codon 232 mutation of PRNP. Neurology. 59:1619-1621

39. Landrum MJ, Lee JM, Benson M, Brown G, Chao C, Chitipiralla S et al (2016) ClinVar: public archive of interpretations of clinically relevant variants. Nucleic Acids Res. 44:D862-D868

40. Le Ber I, Camuzat A, Hannequin D, Pasquier F, Guedj E, Rovelet-Lecrux A et al (2008) Phenotype variability in progranulin mutation carriers: a clinical, neuropsychological, imaging and genetic study. Brain. 131:732-746

41. Lek M, Karczewski KJ, Minikel EV, Samocha KE, Banks E, Fennell T et al (2016) Analysis of protein-coding genetic variation in 60,706 humans. Nature. 536: 285-291

42. Leverenz JB, Yu CE, Montine TJ, Steinbart E, Bekris LM, Zabetian C et al (2007) A novel progranulin mutation associated with variable clinical presentation and tau, TDP43 and alpha-synuclein pathology. Brain. 130: 1360-1374

43. Liem MK, Lesnik SA, Vollebregt MJ, Middelkoop HAM, van der Grond J, den Enden ATJMH (2008) Homozygosity for a NOTCH3 mutation in a 65-yearold CADASIL patient with mild symptoms. J Neurol. Steinkopff-Verlag 255: 1978-1980

44. Liu X, Jian X, Boerwinkle E (2013) dbNSFP v2.0: a database of human nonsynonymous SNVs and their functional predictions and annotations. Hum Mutat. 34:E2393-E2402
45. Mackenzie IR, Nicholson AM, Sarkar M, Messing J, Purice MD, Pottier C et al (2017) TIA1 Mutations in Amyotrophic Lateral Sclerosis and Frontotemporal Dementia Promote Phase Separation and Alter Stress Granule Dynamics. Neuron 95:808-16.e9

46. Maksemous N, Smith RA, Haupt LM, Griffiths LR (2016) Targeted next generation sequencing identifies novel NOTCH3 gene mutations in CADASIL diagnostics patients. Hum Genomics. 10:38

47. Marui W, Iseki E, Kato M, Akatsu H, Kosaka K (2004) Pathological entity of dementia with Lewy bodies and its differentiation from Alzheimer's disease. Acta Neuropathol. 108:121-128

48. McAleese KE, Walker L, Erskine D, Thomas AJ, McKeith IG, Attems J (2017) TDP-43 pathology in Alzheimer's disease, dementia with Lewy bodies and ageing. Brain Pathol. 27:472-479

49. McKeith IG, Dickson DW, Lowe J, Emre M, O'Brien JT, Feldman H et al (2005) Diagnosis and management of dementia with Lewy bodies: third report of the DLB Consortium. Neurology. 65:1863-1872

50. McKenna A, Hanna M, Banks E, Sivachenko A, Cibulskis K, Kernytsky A et al (2010) The Genome Analysis Toolkit: a MapReduce framework for analyzing next-generation DNA sequencing data. Genome Res. 20:1297-1303

51. Meeus B, Verstraeten A, Crosiers D, Engelborghs S, Van den Broeck M, Mattheijssens $M$ et al (2012) DLB and PDD: a role for mutations in dementia and Parkinson disease genes? Neurobiol Aging 33:629.e5-629.e18

52. Nagahama Y, Fukui T (2019) Dementia with Lewy bodies presenting as frontotemporal dementia phenotype. Psychogeriatrics. Available from: https://doi.org/10.1111/psyg.12405

53. Nalls MA, Duran R, Lopez G, Kurzawa-Akanbi M, McKeith IG, Chinnery PF et al (2013) A multicenter study of glucocerebrosidase mutations in dementia with Lewy bodies. JAMA Neurol. 70:727-735

54. Ogaki K, Koga S, Heckman MG, Fiesel FC, Ando M, Labbé C et al (2015) Mitochondrial targeting sequence variants of the $\mathrm{CHCHD} 2$ gene are a risk for Lewy body disorders. Neurology. 85:2016-2025

55. Ohtake H, Limprasert P, Fan Y, Onodera O, Kakita A, Takahashi H et al (2004) Beta-synuclein gene alterations in dementia with Lewy bodies. Neurology. 63:805-811

56. Peacock ML, Warren JT Jr, Roses AD, Fink JK (1993) Novel polymorphism in the A4 region of the amyloid precursor protein gene in a patient without Alzheimer's disease. Neurology. 43:1254-1256

57. Pickering-Brown SM, Mann DM, Bourke JP, Roberts DA, Balderson D, Burns A et al (1994) Apolipoprotein E4 and Alzheimer's disease pathology in Lewy body disease and in other beta-amyloid-forming diseases. Lancet. 343:1155

58. Purcell S, Neale B, Todd-Brown K, Thomas L, Ferreira MAR, Bender D et al (2007) PLINK: a tool set for whole-genome association and populationbased linkage analyses. Am J Hum Genet. 81:559-575

59. Rademakers R, Baker M, Gass J, Adamson J, Huey ED, Momeni P et al (2007) Phenotypic variability associated with progranulin haploinsufficiency in patients with the common $1477 \mathrm{C} \rightarrow \mathrm{T}($ Arg493X) mutation: an international initiative. Lancet Neurol. 6:857-868

60. Revuelta GJ, Rosso A, Lippa CF (2008) Association Between Progranulin and ß-Amyloid in Dementia With Lewy Bodies. Am J Alzheimers Dis Other Demen 23:488-493

61. Sekiguchi H, Moriwaki M, Iritani S, Habuchi C, Torii Y, Umeda K et al (2017) An autopsy case of dementia with Lewy bodies clinically diagnosed to have a behavioral variant of frontotemporal dementia. Clin Neuropathol. 2017(36):23-30

62. Sims R, van der Lee SJ, Naj AC, Bellenguez C, Badarinarayan N, Jakobsdottir $J$ et al (2017) Rare coding variants in PLCG2, ABI3, and TREM2 implicate microglial-mediated innate immunity in Alzheimer's disease. Nat Genet. 49: $1373-1384$

63. Singleton AB, Farrer $M$, Johnson J, Singleton A, Hague S, Kachergus J et al (2003) alpha-Synuclein locus triplication causes Parkinson's disease. Science 302:841

64. Singleton AB, Wharton A, O'Brien KK, Walker MP, McKeith IG, Ballard CG et al (2002) Clinical and neuropathological correlates of apolipoprotein $E$ genotype in dementia with Lewy bodies. Dement Geriatr Cogn Disord. 14: 167-175

65. Stanford PM, Brooks WS, Teber ET, Hallupp M, McLean C, Halliday GM et al (2004) Frequency of tau mutations in familial and sporadic frontotemporal dementia and other tauopathies. J Neurol. 251:1098-1104

66. Stenson PD, Mort M, Ball EV, Howells K, Phillips AD, Thomas NS et al (2009) The Human Gene Mutation Database: 2008 update. Genome Med. 1:13

67. Testi S, Malerba G, Ferrarini M, Ragno M, Pradotto L, Mauro A et al (2012) Mutational and haplotype map of NOTCH3 in a cohort of Italian patients 
with cerebral autosomal dominant arteriopathy with subcortical infarcts and leukoencephalopathy (CADASIL). J Neurol Sci. 319:37-41

68. Tikka S, Mykkänen K, Ruchoux M-M, Bergholm R, Junna M, Pöyhönen M et al (2009) Congruence between NOTCH3 mutations and GOM in 131 CADASIL patients. Brain. 132:933-939

69. Tsuang D, Leverenz JB, Lopez OL, Hamilton RL, Bennett DA, Schneider JA et al (2013) APOE 4 Increases Risk for Dementia in Pure Synucleinopathies. JAMA Neurol 70:223-228

70. Van der Auwera GA, Carneiro MO, Hartl C, Poplin R, Del Angel G, LevyMoonshine A et al (2013) From FastQ data to high confidence variant calls: the Genome Analysis Toolkit best practices pipeline. Curr Protoc Bioinformatics. 43:11.10.1-11.1033

71. van der Lee SJ, Conway OJ, Jansen I, Carrasquillo MM, Kleineidam L, van den Akker E et al (2019) A nonsynonymous mutation in PLCG2 reduces the risk of Alzheimer's disease, dementia with Lewy bodies and frontotemporal dementia, and increases the likelihood of longevity. Acta Neuropathol. 138: 237-250

72. Van Mossevelde S, Engelborghs S, van der Zee J, Van Broeckhoven C. Genotype-phenotype links in frontotemporal lobar degeneration. Nat Rev Neurol. 2018;14:363-378. Available from: doi: https://doi.org/10.1038/s41582018-0009-8

73. van Swieten JC, Heutink P (2008) Mutations in progranulin (GRN) within the spectrum of clinical and pathological phenotypes of frontotemporal dementia. Lancet Neurol. 7:965-974

74. Whiffin N, Minikel E, Walsh R, O'Donnell-Luria AH, Karczewski K, Ing AY et al (2017) Using high-resolution variant frequencies to empower clinical genome interpretation. Genet Med. 19:1151-1158

75. Wu Q, Chen M, Buchwald M, Phillips RA (1995) A simple, rapid method for isolation of high quality genomic DNA from animal tissues. Nucleic Acids Res. 23:5087-5088

76. Yu C-E, Bird TD, Bekris LM, Montine TJ, Leverenz JB, Steinbart E et al (2010) The spectrum of mutations in progranulin: a collaborative study screening 545 cases of neurodegeneration. Arch Neurol. 67:161-170

77. Zarranz JJ, Alegre J, Gómez-Esteban JC, Lezcano E, Ros R, Ampuero I et al (2004) The new mutation, E46K, of alpha-synuclein causes Parkinson and Lewy body dementia. Ann Neurol. 55:164-173

\section{Publisher's Note}

Springer Nature remains neutral with regard to jurisdictional claims in published maps and institutional affiliations.

Ready to submit your research? Choose BMC and benefit from:

- fast, convenient online submission

- thorough peer review by experienced researchers in your field

- rapid publication on acceptance

- support for research data, including large and complex data types

- gold Open Access which fosters wider collaboration and increased citations

- maximum visibility for your research: over $100 \mathrm{M}$ website views per year

At $\mathrm{BMC}$, research is always in progress.

Learn more biomedcentral.com/submissions 